\title{
Testosterone improves erectile function through inhibition of reactive oxygen species generation in castrated rats
}

Rui Li, Xianghu Meng, Yan Zhang, Tao Wang, Jun Yang, Yonghua Niu, Kai Cui, Shaogang Wang, Jihong Liu, Ke Rao

Testosterone is overwhelmingly important in regulating erectile physiology. However, the associated molecular mechanisms are poorly understood. The purpose of this study was to explore the effects and mechanism of testosterone in erectile dysfunction (ED) in castrated rats. Forty male Sprague-Dawley rats were randomized to 4 groups (control, shamoperated, castration and castration-with-testosterone-replacement). Reactive oxygen species (ROS) production was measured by dihydroethidium (DHE) staining. Erectile function was assessed by the recording of intracavernous pressure (ICP) and mean arterial blood pressure (MAP). Protein expression levels were examined by Western blotting. We found that castration reduced erectile function and that testosterone restored it. Nitric oxide synthase (NOS) activity was decrease in the castrated rats, and testosterone administration attenuated this decrease (each $p<0.05$ ). The testosterone, dihydrotestosterone, cyclic guanosine monophosphate (cGMP) and cyclic adenosine monophosphate (CAMP) concentrations were lower in the castrated rats, and testosterone restored these levels (each $p<0.05)$. Furthermore, the cyclooxygenase-2 (COX-2) and prostaglandin synthase (PTGIS) expression levels and phospho-endothelial nitric oxide synthase ( $p$-eNOS, Ser1177)/endothelial nitric oxide synthase (eNOS) ratio were reduced in the castrated rats compared with the controls (each $p<0.05$ ). In addition, the $p 40^{\text {phox }}$ and p67 $7^{\text {phox }}$ expression levels were increased in the castrated rats, and testosterone reversed these changes (each $p<0.05$ ). Overall, our results demonstrate that testosterone ameliorates ED after castration by reducing ROS production and increasing the activity of the eNOS/cGMP and COX-2/PTGIS/CAMP signaling pathways. 


\section{Testosterone Improves Erectile Function through the}

2 Inhibition of Reactive Oxygen Species Generation in

3 Castrated Rats

5 Running Title: Testosterone Improves Erectile Function in Castrated Rats

7 Rui Li $^{1,2}$, Xianghu Meng ${ }^{1,2}$, Yan Zhang ${ }^{1,2}$, Tao Wang ${ }^{1,2}$, Jun Yang ${ }^{1,2}$, Yonghua Niu ${ }^{1,2}$, Kai Cui ${ }^{1,2}$,

8 Shaogang Wang ${ }^{1,2}$, Jihong $\operatorname{Liu}^{1,2}, \mathrm{Ke}^{\mathrm{Rao}}{ }^{1,2}$

101 Department of Urology, Tongji Hospital, Tongji Medical College, Huazhong University of

11 Science and Technology, Wuhan, 430030, Hubei, China.

122 Institute of Urology, Tongji Hospital, Tongji Medical College, Huazhong University of

13 Science and Technology, Wuhan, 430030, Hubei, China.

15 Corresponding Authors:

16 Jihong Liu, MD, PhD, Department of Urology, Institute of Urology, Tongji Hospital, Tongji

17 Medical College, Huazhong University of Science and Technology, Wuhan, 430030, Hubei,

18 China. E-mail: jhliu@tjh.tjmu.edu.cn; Tel.: (86) 027-83663460; Fax: (86) 027-83663460;

19 Ke Rao, MD, PhD, Department of Urology, Institute of Urology, Tongji Hospital, Tongji

20 Medical College, Huazhong University of Science and Technology, Wuhan, 430030, Hubei,

21 China. E-mail: raokeke2009@163.com; Tel.: (86)027-83663460; Fax: (86) 027-83663460. 
$22 *$ Current address: Department of Urology, First Affiliated Hospital of Nanjing Medical

23 University, Nanjing, 210029, Jiangsu, China. 


\section{5 \\ INTRODUCTION}

26 Testosterone is overwhelmingly important in regulating erectile physiology through various

27 signaling pathways (Bond et al. 2010; Chua et al. 2009; Zhang et al. 2011b). Erectile dysfunction

28 (ED) is a common symptom in hypogonadal men that can lead to decreased self-confidence, depression and other symptoms that seriously influence the quality of life (Yohannes et al. 2010).

A recent study has revealed that testosterone yields many benefits in the treatment of

hypogonadism and ED (Yassin et al. 2014). However, the mechanism of how testosterone improves ED is not completely understood.

soluble guanylyl cyclase and the accumulation of cyclic guanosine monophosphate (cGMP), resulting in the relaxation of smooth muscle and penile erection (Andersson \& Wagner 1995;

or Rac2, is a crucial enzyme that catalyzes the production of reactive oxygen species (ROS).

Recent studies have reported that ROS play a major role in hypercholesterolemia-induced ED and diabetes-related ED pathogenesis (Jin et al. 2008; Li et al. 2012; Musicki et al. 2010; Yang et al. 2013). During the process of hypercholesterolemia-induced and diabetes-related ED, increased oxidative stress leads to an imbalance between the limited antioxidant defenses and accumulated ROS, which induces endothelial dysfunction and decreases NO availability. Finally, increased oxidative stress from the NO/cGMP signaling pathway causes pathological ED 
46 signaling pathway have been documented over the last several years, to the best of our

47 knowledge, no comparative studies have been performed on the role of testosterone in

48

ameliorating ROS in castration-induced ED. Hence, we hypothesized that the above-mentioned changes are present in castrated rats and that testosterone improves erectile function by inhibiting ROS production.

Reduction of the cyclic adenosine monophosphate (cAMP) concentration also occurs in ED. Cyclooxygenase (COX) is an important enzyme involved in prostaglandin synthesis; COX-1 and COX-2 are two COX isoforms. COX-1 is constitutively expressed in cells, and COX-2 is expressed under certain anomalous conditions (Wang et al. 2014). Both of these isoforms transform arachidonic acid into prostaglandin $\mathrm{H}_{2}\left(\mathrm{PGH}_{2}\right)$, which is further converted into prostaglandin $\mathrm{I}_{2}$ by prostaglandin synthase (PTGIS). Then, adenylyl cyclase is sensitized to produce cAMP. This activation causes smooth muscle relaxation and penile erection (Lin et al. 2013). $\mathrm{PGH}_{2}$ can also be converted into other prostaglandins with potent proinflammatory effects. Any factors affecting this pathway and leading to cAMP reduction may cause ED. Israel PérezTorres et al have found that castration influences arachidonic acid metabolism and reduces COX2 expression in the kidneys of metabolic syndrome rats (Fernandez-Sanchez et al. 2011).

Similarly, Lin et al have suggested that COX-2-10aa-PGIS gene therapy elevates erectile function following cavernous nerve injury to rats (Lin et al. 2013). However, the role of the COX-2/PTGIS/cAMP signaling pathway remains to be elucidated in castrated rats with ED. The purpose of this study was to determine the effect of testosterone on the erection process in castrated rats. We analyzed the function of testosterone and investigated the molecular 
67 mechanisms of castration-induced ED. 
69 MATERIALS AND METHODS

70 Castration Model and Treatment

71 In the experiment, 40 adult male, 8-week-old Sprague-Dawley rats weighing 200 250 g were

72

obtained from Tongji Medical College, Huazhong University of Science and Technology. The rats were randomized into the following 4 groups: control, sham-operated, surgical castration, and castration-with-testosterone-replacement ( $\mathrm{n}=10$ for each group). The castration procedure was as follows. Briefly, the rats were anesthetized with pentobarbital sodium intraperitoneally (40 $\mathrm{m} \mathrm{kg}^{-1}$ ). A ventral midline incision was created above the scrotum, and the abdominal wall was cut open. The spermatic cord was then separated, and the vas deferens and associated vasculature were identified and separately ligated. Next, the testicles were removed bilaterally. The rats in the testosterone treatment groups received $100 \mathrm{mg} \mathrm{kg}^{-1} \mathrm{month}^{-1}$ testosterone (Zhejiang Xianju Pharmaceutical Co., Ltd., Taizhou, Zhejiang, China, subcutaneous injection) for 1 month immediately after castration (Zhang et al. 2011a). All procedures involving animals were performed in accordance with the guidelines of the Chinese Council on Animal Care and with approval from the Committees on Animal Experiments at Tongji Hospital (Tongji Medical College, Huazhong University of Science and Technology, Wuhan, Hubei, China; ID: TJA20131213).

\section{In Vivo Assessment of Erectile Function}

Erectile function was assessed in all rats after 1 month of testosterone treatment. The assessments were conducted as previously described ( $\mathrm{Li}$ et al. 2012). First, the cavernous nerves were exposed and mounted onto stainless steel bipolar wire electrodes, which were connected to 
90

91

92

93

an electrical stimulator. The electrical stimulation parameters were as follows: 5 volts at $15 \mathrm{~Hz}$, with a square-wave duration of $1.2 \mathrm{~ms}$ for $1 \mathrm{~min}$. Then, a PE-50 cannula (Becton Dickinson \& Co., Sparks, MD, USA) was inserted into the left carotid artery to monitor the systemic mean arterial blood pressure (MAP). Finally, a 25-gauge needle was inserted at the crura, connected to PE-50 tubing, and filled with $250 \mathrm{U} \mathrm{mL}^{-1}$ of a heparin solution. Both blood pressure and intracavernous pressure (ICP) were measured continuously using a data acquisition system (AD Instruments Powerlab/4SP, Bella Vista, NSW, Australia). The Max ICP/MAP was recorded for each rat. The animals were sacrificed via injection of $20 \mathrm{~mL}$ of air, and the corporeal tissue was immediately collected from each rat. One-third of the sample was fixed in $4 \%$ triformol and embedded in paraffin for further use. The remaining tissue was immediately frozen and stored at $-80^{\circ} \mathrm{C}$ until analysis.

\section{Measurements of Plasma Testosterone and Dihydrotestosterone (DHT)}

Immediately after electrostimulation, blood was collected using a PE-50 tube, which was inserted into the left carotid artery, to determine the testosterone and DHT levels. Whole blood was centrifuged at $1580 \mathrm{~g}$ for $15 \mathrm{~min}$ at $4^{\circ} \mathrm{C}$. The testosterone level was determined at the clinical laboratory of Tongji Hospital. The DHT level was determined using an ELISA kit (Westang Bio-tech Co., Ltd., Shanghai, China ). The remaining plasma was collected and stored at $-80^{\circ} \mathrm{C}$.

\section{SDS-PAGE and Immunoblotting}

The frozen penile tissues were prepared in ice-cold RIPA buffer containing a protease inhibitor cocktail and sodium fluoride $(\mathrm{NaF})$, followed by centrifugation at $12000 \mathrm{~g}$ for $15 \mathrm{~min}$ at $4^{\circ} \mathrm{C}$. 
111 Protein concentrations were assayed using a BCA assay kit (Beyotime Institute of Biotechnology,

112 Haimen, Jiangsu, China). In total, $50 \mu \mathrm{g}$ of protein was loaded onto a $10 \%$ sodium dodecyl

113 sulfate-polyacrylamide precast gel and then transferred to a polyvinylidene fluoride membrane.

114 The membranes were blocked for $1 \mathrm{~h}$ in a solution of $0.1 \%$ Tris-buffered saline and Tween-20

115 (TBST) with 5\% (w/v) bovine serum albumin at room temperature. The membranes were

116 subsequently incubated with antibodies against p40phox (1:500, Bioworld, Nanjing, Jiangsu,

117 China), p67phox (1:1000, Affinity, Zhenjiang, Jiangsu, China), endothelial nitric oxide synthase

118 (eNOS, 1:1000, Abcam, Cambridge, MA, USA), phospho-eNOS at Ser1177 (p-eNOS, 1:1000,

119 Cell Signal Technology, Beverly, MA, USA), COX-2 (1:500, Abcam, Hong Kong, China),

PTGIS (1:1000, Abcam, Hong Kong, China) or $\beta$-actin (1:500, Multisciences, Hangzhou,

Zhejiang, China) overnight at $4^{\circ} \mathrm{C}$. After the membranes were washed three times in TBST for 1

$\mathrm{h}$, they were incubated with a secondary antibody in TBST at room temperature for $1.5 \mathrm{~h}$. Then,

they were washed again three times in TBST and analyzed with an enhanced chemiluminescence

detection system (Pierce, Thermo Fisher Scientific, Rockford, IL, USA).

\section{Detection of ROS}

The rat corpora cavernosa were quickly frozen, cut to a thickness of $8 \mu \mathrm{m}$ at an optimized cutting temperature, and placed on glass slides. A fresh dihydroethidium (DHE) solution $\left(1 \mu \mathrm{mol} \mathrm{L} \mathrm{L}^{-1}\right.$,

Beyotime Institute of Biotechnology, Haimen, Jiangsu, China) was topically applied to each tissue slice, and the slices were incubated for $30 \mathrm{~min}$ at $37^{\circ} \mathrm{C}$ in the dark. Fluorescence images were captured with an Olympus BX51 fluorescence microscope (Olympus Corporation, Tokyo, Japan). Fluorescence intensities were determined using Image-Pro Plus software (Media 
132 Cybernetics Inc., Bethesda, MD, USA).

133

134

135

136

137

138

139

140

141

142

143

144

145

146

147

\section{Determination of Nitric Oxide Synthase (NOS) Activity}

NOS activity in the penile tissues was measured using an ELISA kit (Nanjing Jiancheng

Bioengineering Institute, Nanjing, Jiangsu, China) according to the manufacturer's instructions.

The assays were performed in duplicate, and the protein concentrations were detected to normalize the data.

\section{cGMP and cAMP Concentrations}

The cGMP and cAMP concentrations in the penile tissues were measured using an ELISA kit

(Nanjing Jiancheng Bioengineering Institute, Nanjing, Jiangsu, China) according to the

manufacturer's instructions. The assays were performed in duplicate, and the protein

concentrations were detected to normalize the data.

\section{Statistical Analysis}

Parametric data are expressed as the mean \pm SD. All statistical analyses were performed with

SPSS 15.0 software (SPSS, Inc., Chicago, IL, USA) using one-way ANOVA followed by

Bonferroni's multiple comparison post-test. Intergroup differences were considered significant at a $p<0.05$. 
149

150

151

152

153

154

155

156

157

158

159

160

161

162

163

164

165

166

167

168

169

\section{RESULTS}

\section{Effect of Testosterone Treatment on Plasma Testosterone and DHT Concentrations}

The castrated rats exhibited marked decreases in body weight, plasma testosterone and DHT levels compared with the control rats. Testosterone replacement restored the testosterone and DHT concentrations, but they were still lower than those of the control rats (although this difference was not significant). There were no differences in the plasma testosterone and DHT concentrations between the control and sham-operated rats (Table 1).

\section{Effects of Testosterone Treatment on Erectile Function}

Fig. 1 presents a summary of the Max ICP/MAP ratios for the four groups. The Max ICP/MAP ratio was lower in the castrated group than in the other three groups subjected to $5 \mathrm{~V}$ stimulation. Testosterone therapy resulted in a substantial increase in the Max ICP/MAP ratio compared with that of the castration group with electrostimulation $(p<0.05)$. However, this ratio was still lower than those of the control and sham-operated rats. There was no difference in the MAP among the four groups.

\section{Effects of Testosterone Treatment on ROS Production in Penile Cavernous Tissue}

ROS production was detected in the four groups. As shown in Fig. 2A and 2B, castration resulted in a dramatic increase in ROS production (detected by DHE fluorescence), which was attenuated by testosterone. Furthermore, to assess whether castration-induced ROS in the corpus cavernosum is associated with NADPH oxidase, the protein expression levels of the NADPH oxidase subunits $\mathrm{p} 40^{\text {phox }}$ and $\mathrm{p} 67^{\text {phox }}$ were analyzed. Western blot analysis indicated that these levels were greatly increased in the castrated rats compared with the control and sham-operated 
170

171

172

173

174

175

176

177

178

179

180

rats and that they were markedly reduced by testosterone treatment $(p<0.05$, Fig. 2C, 2D, 2E).

\section{Effects of Testosterone Treatment on the NOS/cGMP Signaling Pathways in Penile}

\section{Cavernous Tissue}

The expression levels of eNOS and p-eNOS (Ser1177) in the corpus cavernosum were measured by Western blotting. The p-eNOS (Ser1177)/eNOS ratio was substantially lower in castrated rats than that in the normal control rats. Treatment with testosterone significantly increased the $\mathrm{p}$ eNOS (Ser1177)/eNOS ratio in the castrated rats ( $p<0.05$, Fig. 3A, 3B). In addition, to confirm the bioavailability of NO, ELISAs were performed to assess the cavernous NOS activity and cGMP concentration. As shown in Fig. 3C and 3D, the cavernous NOS activity and cGMP concentration were markedly lower in the castrated rats compared with the control and shamoperated rats (each $p<0.05$ ), indicating that the cGMP-protein-kinase-G axis mediated this inhibitory effect of NO. Testosterone treatment significantly attenuated the castration-induced reduction in cavernous cGMP and NOS activity $(p<0.05)$.

\section{Effects of Testosterone Treatment on the COX-2/cAMP Signaling Pathway in Penile}

\section{Cavernous Tissue}

The cavernous COX-2 and PTGIS protein expression levels were determined in the four groups.

These levels were significantly lower in the castration group than in the control and shamoperated groups (each $p<0.05$ ), and they were increased after 1 month of testosterone treatment (each $p<0.05$, Fig. 4A, 4C, 4D). Further, the cAMP concentration was significantly lower in the penile tissue of the castrated rats compared with those of the control and sham-operated rats ( $p<$ 0.05 , Fig. 4B). The testosterone treatment strongly inhibited the castration-induced reduction in 
191 cavernous cAMP ( $p<0.05$, Fig. 4B). 
193

194 195

196

197

198

199

200

201

202

203

204

205

206

207

208

209

210

211

212

213

\section{DISCUSSION}

Testosterone replacement therapy has been widely studied and has been clinically used for treatment of ED. However, the underlying molecular mechanisms of exogenous testosterone administration are not fully understood and are worthy of a detailed study. ROS play important roles in various diseases, including cancer, obesity, and ED

(Fernandez-Sanchez et al. 2011; Raj et al. 2011; Silva et al. 2014), via reactive elements

produced by the reduction of $\mathrm{O}_{2}$ with a single electron (superoxide), two electrons (hydrogen peroxide) or three electrons (hydroxyl radical) (Sabharwal \& Schumacker 2014). A recent study

has reported that the penile ROS levels are significantly increased and that eNOS/cGMP activities are reduced in diabetes-related ED (Yang et al. 2013). However, no correlative studies have been performed using a castrated rat model. Excessive ROS production or the failure of oxidant cleaning systems can obstruct cellular function through the oxidation of proteins, lipids and DNA (Murphy et al. 2011). In our study, we found that the levels of ROS were obviously increased and that those of the NADPH oxidase subunits $\mathrm{p} 40^{\text {phox }}$ and $\mathrm{p} 67^{\text {phox }}$ were also increased in the castrated rat model. The up-regulation of $\mathrm{p} 40^{\mathrm{phox}}$ and $\mathrm{p} 67^{\text {phox }}$ resulted in increased ROS levels in the corpus cavernosum. Therefore, the increased production of ROS, which are activated by enzymes involved in their shape (especially NADPH oxidase), might be a key mechanism underlying castration-induced ED.

Several studies have revealed that testosterone is crucial for exerting antioxidant effects by decreasing ROS. Hwang et al have demonstrated that testosterone supplementation reduces oxidative damage in Leydig cells (Hwang et al. 2011). However, the effect of testosterone on 
214 ROS levels in the corpora cavernosa of castrated rats is still unclear and needs to be clarified. We

215 found that testosterone treatment reduced ROS level and $\mathrm{p} 40^{\mathrm{phox}}$ and $\mathrm{p} 67^{\text {phox }}$ expression and

216 improved erectile function. The decrease in NADPH oxidase led to a reduction in ROS. Thus,

217 preventing the generation of ROS by interfering with the enzymes that produce them, especially

218 NADPH oxidase, may be a more valid measure for combating oxidative stress than eliminating

219 ROS after their formation.

The NOS/cGMP pathway, which is the primary erectile pathway, has been shown to be

associated with androgen. A recent study has revealed that low testosterone levels in men are

associated with impaired endothelial function and NO bioavailability (Corrigan et al. 2015; Novo

et al. 2015). Effects of testosterone on the expression of NOS isoforms have been shown in

penile tissue (Lugg et al. 1995; Seo et al. 1999; Traish et al. 2007). Replacement of 5 $\alpha$-DHT and

testosterone has been shown to restore erectile function and NOS expression in the corpus

cavernosum of castrated animals (Schirar et al. 1997; Traish et al. 2007). However, the manner

by which testosterone enhances the activity of the NOS/cGMP pathway is not fully understood.

In this study, we discovered that p-eNOS (Ser1177)/eNOS ratio and the testosterone and cGMP

concentrations were reduced in the castrated rats and that treatment with testosterone restored

these levels. Numerous studies have concluded that increased ROS generation is one of the

major causes of decreased NO bioavailability (De Young et al. 2004; Jin et al. 2009; ZS 1996).

Hence, according to our findings regarding ROS and NADPH oxidase, we believe that treatment

with testosterone ameliorates ED by reducing the expression of the NADPH oxidase subunits p40 phox and 67 $^{\text {phox }}$. These reductions subsequently trigger a decrease in ROS, improvement in 
235

236

237

238

239

240

241

242

endothelial cell function and an increase in NO. Subsequently, these changes lead to an increase in the cGMP concentration and smooth muscle relaxation in the corpus cavernosum.

In males, testosterone is essential for fertility, puberty, sexual motivation, and sexual performance (JJ 2010). Testosterone production is predominantly regulated through the interaction of luteinizing hormone/human chorionic gonadotropin with specific receptors (Catt \& Dufau 1973; ML 1998), resulting in an increased intracellular cAMP level. Recent studies have indicated that cAMP plays an important role in erectile physiology through the COX-2 pathway (Lin et al. 2013; Moreland et al. 2001). COX-2 and PTGIS, which regulate the production of inflammatory mediators, are key enzymes involved in cAMP activation. Prostaglandin E, the formation of which is catalyzed by COX-2 and PTGIS, binds to pathognostic receptors on smooth muscle and is thought to enable the relaxation of smooth muscle by activating cAMPdependent pathways. A lack of testosterone decreases the expression of COX-2 and PTGIS, which in turn results in a reduced cAMP level in the corpus cavernosum. Then, the blocking of cAMP-dependent protein kinase (PKA) activation causes dysfunction in the relaxation of smooth muscle and ED. In our study, we found that COX-2 and PTGIS expression levels were reduced in the castrated rats compared with the control rats. Further, the cAMP concentration was lower in the castrated rats than in the age-matched control rats. Treatment with testosterone markedly increased COX-2 and PTGIS expression, as well as cAMP concentration. These results imply that the COX-2/PTGIS/cAMP signaling pathway may participate in another mechanism responsible for castration-induced ED.

The relationship between testosterone and erectile function has not been completely 
256 elucidated. In our study, we revealed that testosterone improved erectile function through

257 inhibition of ROS generation in the castrated rats. These findings could initiate a new line of

258 research in penis physiology and may provide a further scientific basis for the use of testosterone

259 in the management of ED in men with testosterone insufficiency. We hope that these results can

260 be utilized to produce novel therapeutic mechanisms for the treatment of hypogonadal ED.

261 Recent clinical trials suggested a significant improvement in sexual function and ED in

262 hypogonadal men with testosterone treatment (Giltay et al. 2010; Hackett et al. 2013; Khera

263 2009; Zitzmann et al. 2013), however, the relationship between testosterone and erectile function

264 has not been completely elucidated. In our study, we revealed that testosterone improved erectile

265 function through inhibition of ROS generation in the castrated rats. These findings could initiate

266 a new line of research in penis physiology and may provide a further scientific basis for the use

267 of testosterone in the management of ED in men with testosterone insufficiency. We hope that

268 these results can be utilized to produce novel therapeutic mechanisms for the treatment of

269 hypogonadal ED.

This study has a few limitations. The possible involvement of the COX-2/PTGIS/cAMP

271 signaling pathway in castration-induced ED needs to be further verified. In addition, the effects

272 of testosterone were evaluated over the short-term in our study; and its long-term effects must be

273 assessed in future studies. Finally, the lack of knowledge regarding the long-term effects of

274 testosterone has limited its clinical application. 


\section{CONCLUSIONS}

277 In conclusion, testosterone reduced ROS production and increased eNOS expression in the

278 castrated rats. Further, it activated the COX-2/PTGIS/cAMP signaling pathway and increased

279 cAMP production. In addition, it improved erectile function in the castrated rats under the

280 combined actions of the above-mentioned factors. Therefore, this study presents novel findings

281 that provide insights into the molecular mechanisms of castration-induced ED. Further studies

282 are needed to elucidate the precise mechanisms involved.

283

284 
286

287

288

289

290

291

292

293

294

295

296

297

298

299

300

301

302

303

304

305

306

307

308

309

310

311

312

313

314

315

316

317

318

319

320

321

322

323

324

325

\section{REFERENCES}

Andersson KE, and Wagner G. 1995. Physiology of penile erection. Physiol Rev 75:191-236.

Bond CW, Angeloni NL, and Podlasek CA. 2010. Analysis of testosterone effects on sonic hedgehog signaling in juvenile, adolescent and adult sprague dawley rat penis. $J$ Sex Med 7:1116-1125 DOI 10.1111/j.1743-6109.2009.01585.x.

Burnett AL, and Musicki B. 2005. The nitric oxide signaling pathway in the penis. Curr Pharm Des 11:3987-3994.

Catt KJ, and Dufau ML. 1973. Spare gonadotrophin receptors in rat testis. Nat New Biol 244:219-221.

Chua RG, Calenda G, Zhang X, Siragusa J, Tong Y, Tar M, Aydin M, DiSanto ME, Melman A, and Davies KP. 2009. Testosterone regulates erectile function and Vcsa1 expression in the corpora of rats. Mol Cell Endocrinol 303:67-73 DOI 10.1016/j.mce.2009.02.001.

Corrigan FE, 3rd, Al Mheid I, Eapen DJ, Hayek SS, Sher S, Martin GS, and Quyyumi AA. 2015. Low testosterone in men predicts impaired arterial elasticity and microvascular function. Int J Cardiol 194:94-99 DOI 10.1016/j.ijcard.2015.05.065.

De Young L, Yu D, Bateman RM, and Brock GB. 2004. Oxidative stress and antioxidant therapy: Their impact in diabetes-associated erectile dysfunction. Journal of Andrology 25:830-836.

Fernandez-Sanchez A, Madrigal-Santillan E, Bautista M, Esquivel-Soto J, MoralesGonzalez A, Esquivel-Chirino C, Durante-Montiel I, Sanchez-Rivera G, ValadezVega C, and Morales-Gonzalez JA. 2011. Inflammation, oxidative stress, and obesity. Int J Mol Sci 12:3117-3132 DOI 10.3390/ijms12053117.

Giltay EJ, Tishova YA, Mskhalaya GJ, Gooren LJ, Saad F, and Kalinchenko SY. 2010. Effects of testosterone supplementation on depressive symptoms and sexual dysfunction in hypogonadal men with the metabolic syndrome. J Sex Med 7:2572-2582. DOI 10.1111/j.1743-6109.2010.01859.x.

Hackett G, Cole N, Bhartia M, Kennedy D, Raju J, and Wilkinson P. 2013. Testosterone replacement therapy with long-acting testosterone undecanoate improves sexual function and quality-of-life parameters vs. placebo in a population of men with type 2 diabetes. $J$ Sex Med 10:1612-1627. DOI 10.1111/jsm.12146.

Hwang TI, Liao TL, Lin JF, Lin YC, Lee SY, Lai YC, and Kao SH. 2011. Low-dose testosterone treatment decreases oxidative damage in TM3 Leydig cells. Asian J Androl 13:432-437 DOI 10.1038/aja.2010.159.

Jin HR, Kim WJ, Song JS, Choi MJ, Piao S, Shin SH, Tumurbaatar M, Tuvshintur B, Nam MS, Ryu JK, and Suh JK. 2009. Functional and morphologic characterizations of the diabetic mouse corpus cavernosum: comparison of a multiple low-dose and a single high-dose streptozotocin protocols. J Sex Med 6:3289-3304 DOI 10.1111/j.17436109.2009.01464.x.

Jin L, Lagoda G, Leite R, Webb RC, and Burnett AL. 2008. NADPH oxidase activation: a 
mechanism of hypertension-associated erectile dysfunction. $J$ Sex Med 5:544-551 DOI 10.1111/j.1743-6109.2007.00733.x.

JJ H. 2010. Management of erectile dysfunction. Am Fam Physician 81:305-312.

Khera M. 2009. Androgens and Erectile Function: A Case for Early Androgen Use in Postprostatectomy Hypogonadal Men. Journal of Sexual Medicine 6:234-238. DOI 10.1111/j.1743-6109.2008.01159.x.

Li M, Zhuan L, Wang T, Rao K, Yang J, Yang J, Quan W, Liu J, and Ye Z. 2012. Apocynin improves erectile function in diabetic rats through regulation of NADPH oxidase expression. $J$ Sex Med 9:3041-3050 DOI 10.1111/j.1743-6109.2012.02960.x.

Lin H, Yuan J, Ruan KH, Yang W, Zhang J, Dai Y, and Wang R. 2013. COX-2-10aa-PGIS gene therapy improves erectile function in rats after cavernous nerve injury. $J$ Sex Med 10:1476-1487 DOI 10.1111/jsm.12147.

Lue TF. 2000. Erectile dysfunction. $N$ Engl $J$ Med 342:1802-1813 DOI 10.1056/NEJM200006153422407.

Lugg JA, Rajfer J, and Gonzalez-Cadavid NF. 1995. Dihydrotestosterone is the active androgen in the maintenance of nitric oxide-mediated penile erection in the rat. Endocrinology 136:1495-1501 DOI 10.1210/endo.136.4.7534702.

ML D. 1998. The luteinizing hormone receptor. Annu Rev Physiol 60:461-496.

Moreland RB, Albadawi H, Bratton C, Patton G, Goldstein I, Traish A, and Watkins MT. 2001. $\mathrm{O}_{2}$-dependent prostanoid synthesis activates functional PGE receptors on corpus cavernosum smooth muscle. Am J Physiol Heart Circ Physiol 281:H552-558.

Murphy MP, Holmgren A, Larsson NG, Halliwell B, Chang CJ, Kalyanaraman B, Rhee SG, Thornalley PJ, Partridge L, Gems D, Nystrom T, Belousov V, Schumacker PT, and Winterbourn CC. 2011. Unraveling the biological roles of reactive oxygen species. Cell Metab 13:361-366 DOI 10.1016/j.cmet.2011.03.010.

Musicki B, Liu T, Lagoda GA, Strong TD, Sezen SF, Johnson JM, and Burnett AL. 2010. Hypercholesterolemia-induced erectile dysfunction: endothelial nitric oxide synthase (eNOS) uncoupling in the mouse penis by NAD(P)H oxidase. J Sex Med 7:3023-3032 DOI 10.1111/j.1743-6109.2010.01880.x.

Novo S, Iacona R, Bonomo V, Evola V, Corrado E, Di Piazza M, Novo G, and Pavone C. 2015. Erectile dysfunction is associated with low total serum testosterone levels and impaired flow-mediated vasodilation in intermediate risk men according to the framingham risk score. Atherosclerosis 238:415-419 DOI 10.1016/j.atherosclerosis.2014.12.007.

Raj L, Ide T, Gurkar AU, Foley M, Schenone M, Li X, Tolliday NJ, Golub TR, Carr SA, Shamji AF, Stern AM, Mandinova A, Schreiber SL, and Lee SW. 2011. Selective killing of cancer cells by a small molecule targeting the stress response to ROS. Nature 475:231-234 DOI 10.1038/nature10167.

Sabharwal SS, and Schumacker PT. 2014. Mitochondrial ROS in cancer: initiators, amplifiers or an Achilles' heel? Nat Rev Cancer 14:709-721 DOI 10.1038/nrc3803.

Schirar A, Bonnefond C, Meusnier C, and Devinoy E. 1997. Androgens modulate nitric oxide 
synthase messenger ribonucleic acid expression in neurons of the major pelvic ganglion in the rat. Endocrinology 138:3093-3102 DOI 10.1210/endo.138.8.5310.

Seo SI, Kim SW, and Paick JS. 1999. The effects of androgen on penile reflex, erectile response to electrical stimulation and penile NOS activity in the rat. Asian J Androl 1:169-174.

Silva FH, Leiria LO, Alexandre EC, Davel AP, Monica FZ, De Nucci G, and Antunes E. 2014. Prolonged therapy with the soluble guanylyl cyclase activator BAY 60-2770 restores the erectile function in obese mice. $J$ Sex Med 11:2661-2670 DOI $10.1111 /$ jsm. 12682.

Traish AM, Goldstein I, and Kim NN. 2007. Testosterone and erectile function: from basic research to a new clinical paradigm for managing men with androgen insufficiency and erectile dysfunction. Eur Urol 52:54-70 DOI 10.1016/j.eururo.2007.02.034.

Wang Y, Cao R, Wei B, Chai X, Sun D, Guan Y, and Liu XM. 2014. Diallyl disulfide inhibits proliferation and transdifferentiation of lung fibroblasts through induction of cyclooxygenase and synthesis of prostaglandin E(2). Mol Cell Biochem 393:77-87 DOI 10.1007/s11010-014-2048-9.

Yang J, Wang T, Yang J, Rao K, Zhan Y, Chen RB, Liu Z, Li MC, Zhuan L, Zang GH, Guo SM, Xu H, Wang SG, Liu JH, and Ye ZQ. 2013. S-allyl cysteine restores erectile function through inhibition of reactive oxygen species generation in diabetic rats. Andrology 1:487-494 DOI 10.1111/j.2047-2927.2012.00060.x.

Yassin DJ, Doros G, Hammerer PG, and Yassin AA. 2014. Long-term testosterone treatment in elderly men with hypogonadism and erectile dysfunction reduces obesity parameters and improves metabolic syndrome and health-related quality of life. J Sex Med 11:15671576 DOI $10.1111 /$ jsm.12523.

Yohannes E, Chang J, Tar MT, Davies KP, and Chance MR. 2010. Molecular targets for diabetes mellitus-associated erectile dysfunction. Mol Cell Proteomics 9:565-578 DOI 10.1074/mcp.M900286-MCP200.

Zhang MG, Shen ZJ, Zhang CM, Wu W, Gao PJ, Chen SW, and Zhou WL. 2011a. Vasoactive intestinal polypeptide, an erectile neurotransmitter, improves erectile function more significantly in castrated rats than in normal rats. BJU Int 108:440-446 DOI 10.1111/j.1464-410X.2010.09901.X.

Zhang XH, Melman A, and Disanto ME. 2011b. Update on corpus cavernosum smooth muscle contractile pathways in erectile function: a role for testosterone? J Sex Med 8:1865-1879 DOI 10.1111/j.1743-6109.2011.02218.x.

Zitzmann M, Mattern A, Hanisch J, Gooren L, Jones H, and Maggi M. 2013. IPASS: a study on the tolerability and effectiveness of injectable testosterone undecanoate for the treatment of male hypogonadism in a worldwide sample of 1,438 men. J Sex Med 10:579-588. DOI 10.1111/j.1743-6109.2012.02853.x.

ZS K. 1996. Superoxide anion and endothelial regulation of arterial tone. Free Radic Biol Med 20:443-448. 
408 


\section{Table $\mathbf{1}$ (on next page)}

Table 1 Body weight and plasma T, DHT levels in the four groups

$* p<0.05$ vs the castration group; $\# p<0.05$ vs castration-with-testosterone-replacement group. Data were expressed as the mean $\pm \mathrm{SD}$. $\mathrm{Co}=$ control; So=sham-operated; $\mathrm{Ca}=$ castration; $\mathrm{Ct}=$ castration-with-testosterone-replacement; $\mathrm{T}$, testosterone; $\mathrm{DHT}$, dihydrotestosterone; $\mathrm{N}=$ number of analyzed samples. 


\begin{tabular}{|c|c|c|c|c|c|}
\hline \multirow[t]{2}{*}{ Group } & \multirow[t]{2}{*}{$\mathrm{N}$} & \multicolumn{2}{|c|}{ Body weight, g } & \multirow{2}{*}{$\begin{array}{l}\text { Plasma T } \\
(\mathrm{ng} / \mathrm{mL})\end{array}$} & \multirow{2}{*}{$\begin{array}{c}\text { Plasma DHT } \\
(\mathrm{pg} / \mathrm{mL})\end{array}$} \\
\hline & & Initial & Final & & \\
\hline $\mathrm{Co}$ & 10 & $224.7 \pm 4.7$ & $407 \pm 37 * \#$ & $4.18 \pm 0.27^{*}$ & $142.8 \pm 15.8^{*}$ \\
\hline So & 10 & $225.1 \pm 2.5$ & $409 \pm 26^{*} \#$ & $4.06 \pm 0.19 *$ & $141.0 \pm 18.7^{*}$ \\
\hline $\mathrm{Ca}$ & 10 & $224.5 \pm 3.4$ & $340 \pm 39$ & $0.51 \pm 0.09$ & $48.3 \pm 6.0$ \\
\hline $\mathrm{Ct}$ & 10 & $225.4 \pm 2.0$ & $343 \pm 44$ & $3.93 \pm 0.12 *$ & $136.0 \pm 12.9 *$ \\
\hline
\end{tabular}

$3 * p<0.05$ vs the castration group; $\# p<0.05$ vs castration-with-testosterone-replacement group.

4 Data were expressed as the mean $\pm \mathrm{SD} . \mathrm{Co}=$ control; $\mathrm{So}=$ sham-operated; $\mathrm{Ca}=$ castration;

$5 \mathrm{Ct}=$ castration-with-testosterone-replacement; $\mathrm{T}$, testosterone; DHT, dihydrotestosterone; $\mathrm{N}=$ 6 number of analyzed samples. 


\section{1}

Testosterone treatment increased the Max ICP/MAP during electrical stimulation of the cavernous nerve $(5 \mathrm{~V}, 15 \mathrm{~Hz}, 1 \mathrm{~min})$

Figure 1 Testosterone treatment increased the Max ICP/MAP during electrical stimulation of the cavernous nerve $(5 \mathrm{~V}, 15 \mathrm{~Hz}, 1 \mathrm{~min}) .(A, B)$ Representative ICP and MAP tracings in the four groups. Bar graph depicting Max ICP/MAP ratio. The data are expressed as the mean $\pm \mathrm{SD}$ ( $\mathrm{n}=6 \sim 8 \mathrm{rats} /$ group). $\mathrm{Co}=$ control; So = sham-operated; $\mathrm{Ca}=$ castration; $\mathrm{Ct}=$ castration-with-testosterone-replacement. $* \mathrm{p}<0.05$ vs the castration group; \# $p<0.05$ vs the castration-with-testosterone-replacement group. 


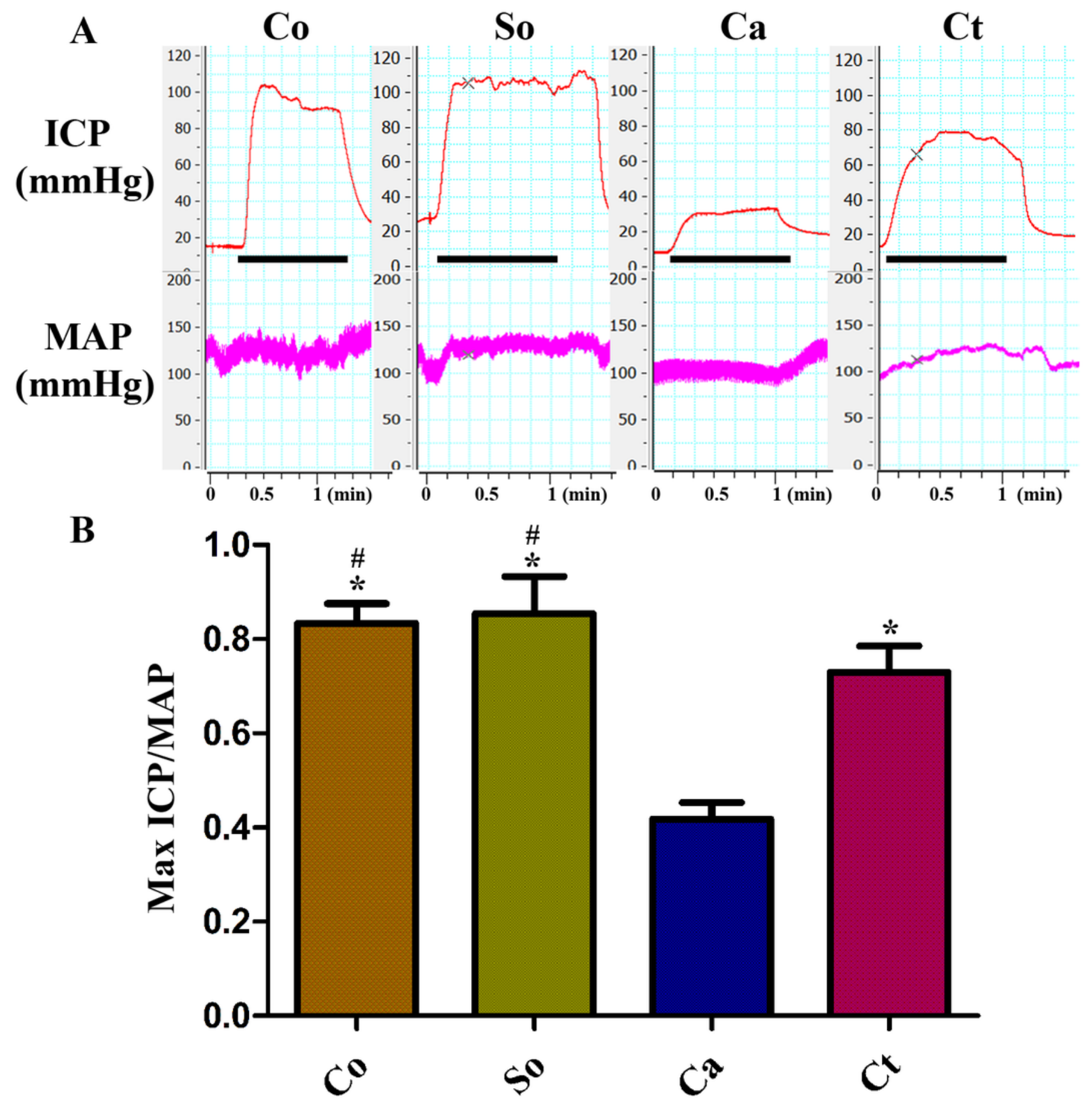


2

Testosterone-induced changes in ROS and protein expression.

Figure 2 Testosterone-induced changes in ROS and protein expression. (A, B) Typical images of DHE in situ staining in corpora cavernosa from rats in the four groups (red fluorescence; scale bars $=100 \mu \mathrm{m}$; time of exposure, $600 \mathrm{~ms}$ ). Red fluorescence intensity was measured using Image-Pro Plus software. (C, D, E) Representative Western blot showing $p 40^{\text {phox }}$ and $p 67^{\text {phox }}$ expression normalized to $\beta$-actin. The data are expressed as the mean \pm $\mathrm{SD}$ ( $\mathrm{n}=6 \sim 9$ rats/group). $\mathrm{Co}=$ control; $\mathrm{So}=$ sham-operated; $\mathrm{Ca}=$ castration; $\mathrm{Ct}=$ castration-with-testosterone-replacement. $* p<0.05$ vs the castration group. ROS, reactive oxygen species; DHE, dihydroethidium. 
A

Co

So

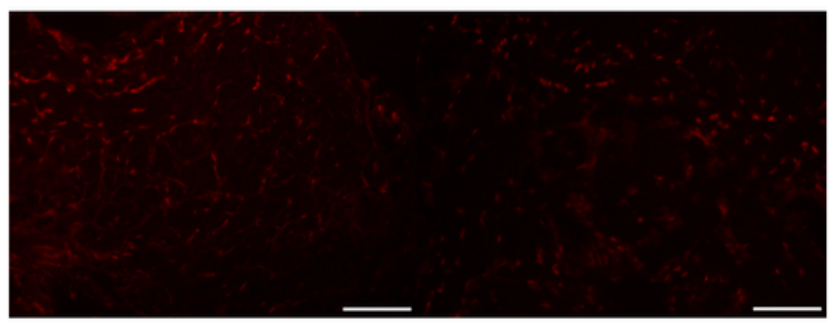

$\mathrm{Ca}$

$\mathrm{Ct}$

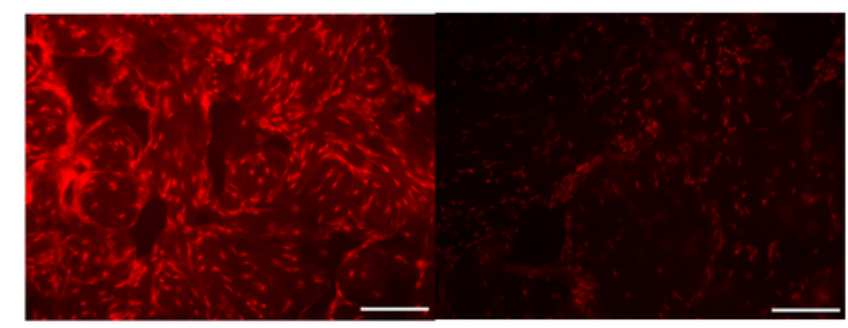

$\mathrm{C}$

Co So $\mathrm{Ca} \quad \mathrm{Ct}$

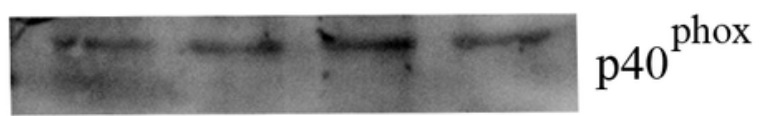

$\mathrm{E}$

$\beta$-actin
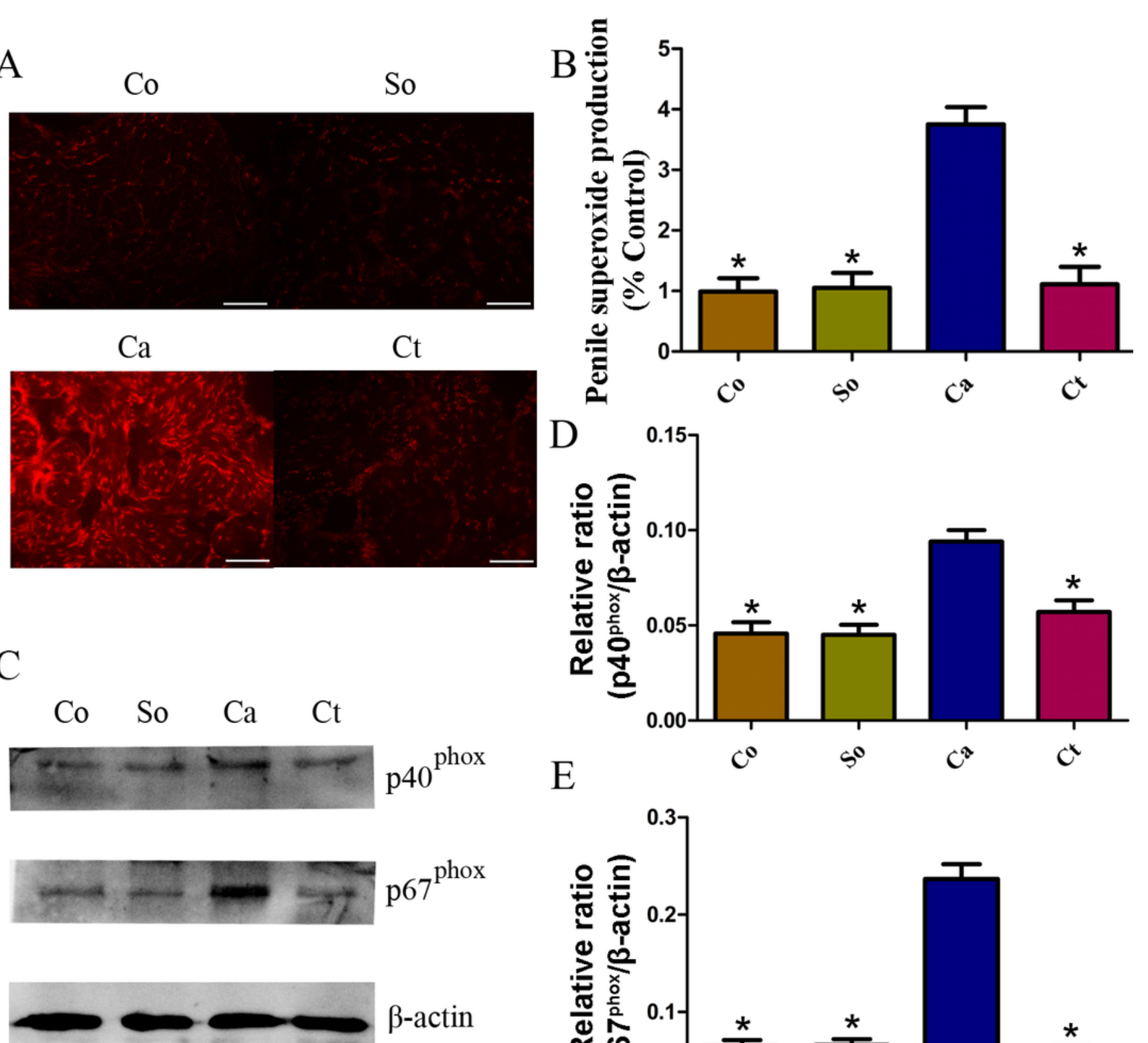

$\mathrm{D}$
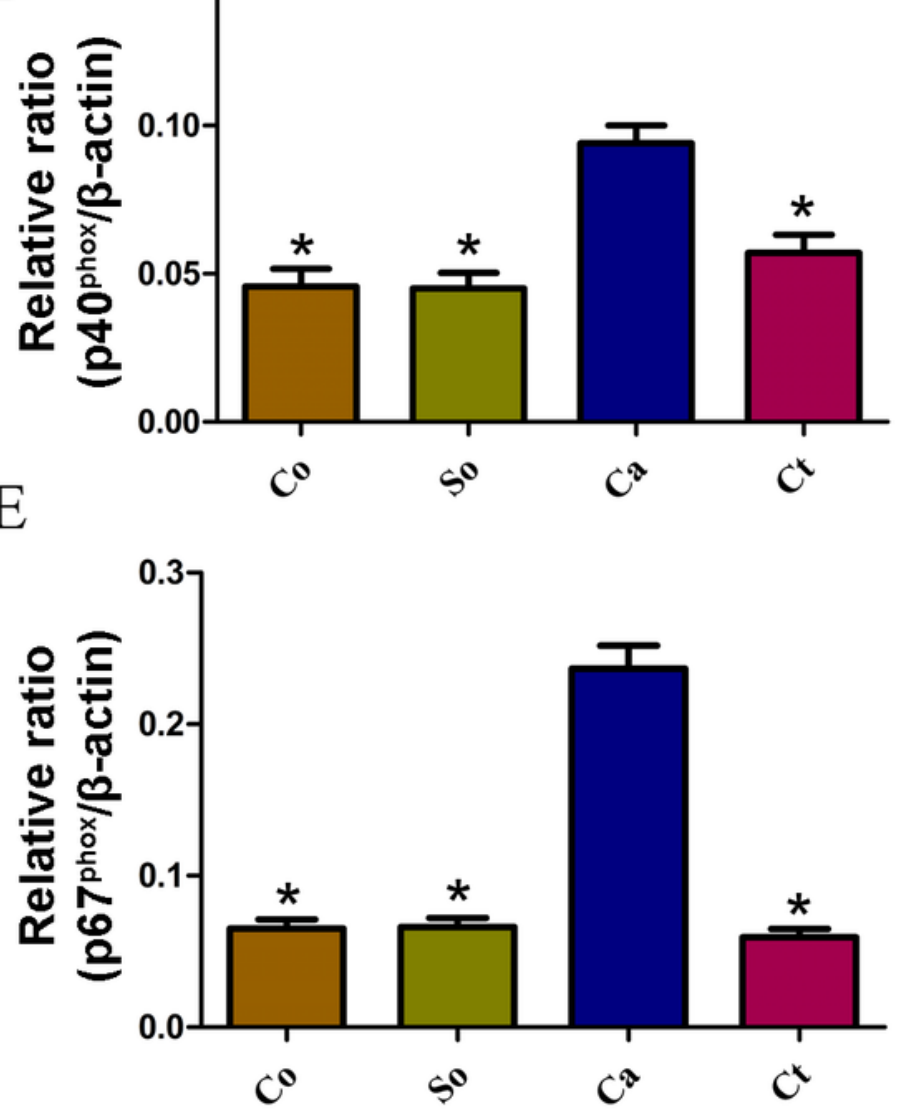


\section{3}

Testosterone-induced increase in the NOS/cGMP signaling pathway in penile tissue

Figure 3 Testosterone-induced increase in the NOS/cGMP signaling pathway in penile tissue. (A, B) Representative Western blotting showing p-eNOS (Ser1177) and eNOS expression normalized to $\beta$-actin, as well as the p-eNOS/eNOS ratio. (C) The cGMP concentration was detected in penile tissue. (D) NOS activity in the four groups. The data are expressed as the mean $\pm S D$ ( $n=6 \sim 9$ rats/group). $C o=$ control; So = sham-operated; $C a=$ castration; $\mathrm{Ct}=$ castration-with-testosterone-replacement. $* \mathrm{p}<0.05 \mathrm{vs}$ the castration group. 


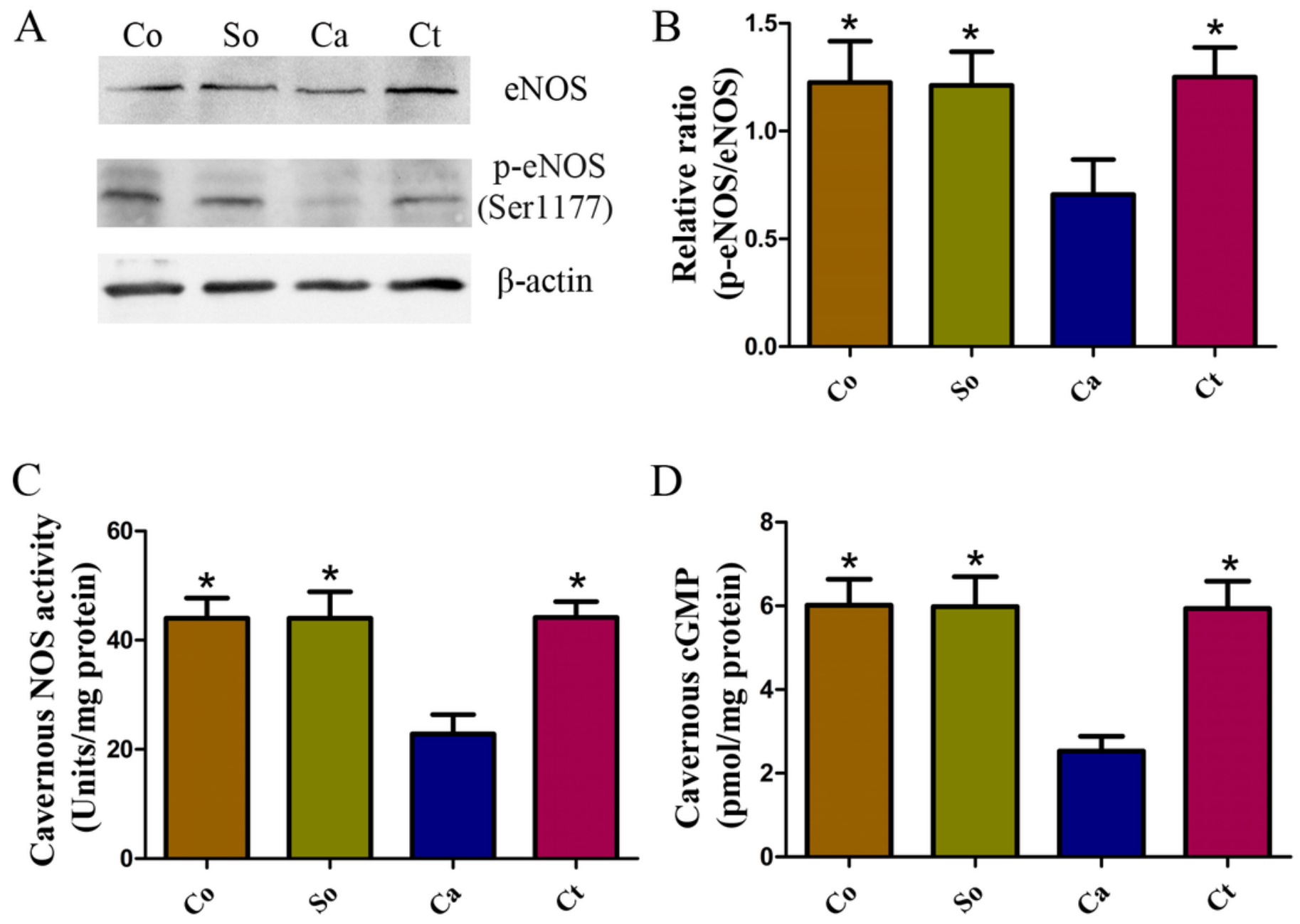




\section{4}

Testosterone-induced increase in the COX-2/CAMP signaling pathway in penile tissue

Figure 4 Testosterone-induced increase in the COX-2/cAMP signaling pathway in penile tissue. (A, C, D) Typical Western blot showing COX-2 and PTGIS protein expression normalized to $\beta$-actin. (B) The CAMP concentration was detected in the penile tissue. The data are expressed as the mean $\pm S D(n=6 \sim 9$ rats/group). Co = control; So = sham-operated; $\mathrm{Ca}=$ castration; $\mathrm{Ct}=$ castration-with-testosterone-replacement. $* \mathrm{p}<0.05$ vs the castration group; $\#<0.05$ vs the castration-with-testosterone-replacement group. 
A B

Co So $\mathrm{Ca} \quad \mathrm{Ct}$

COX-2

$-1-2$ PTGIS

$\beta$-actin

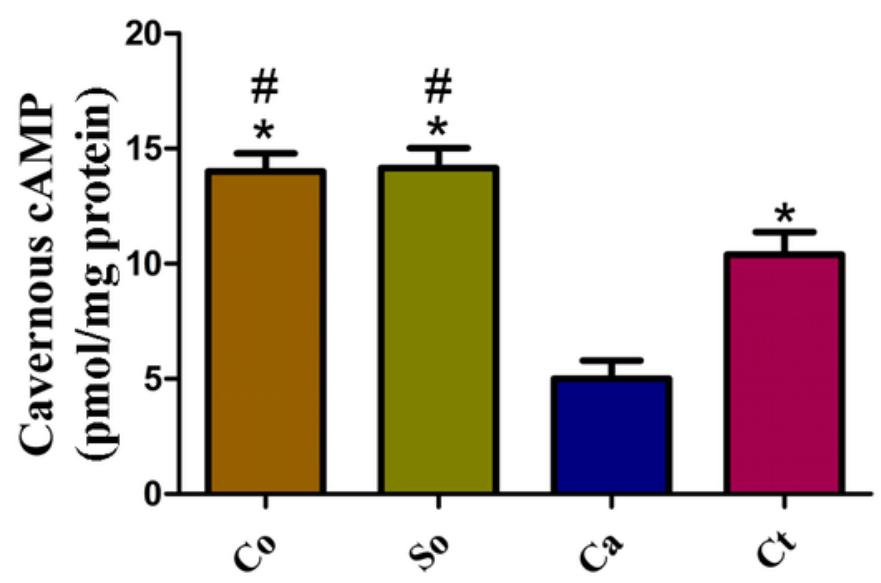

C

$\mathrm{D}$
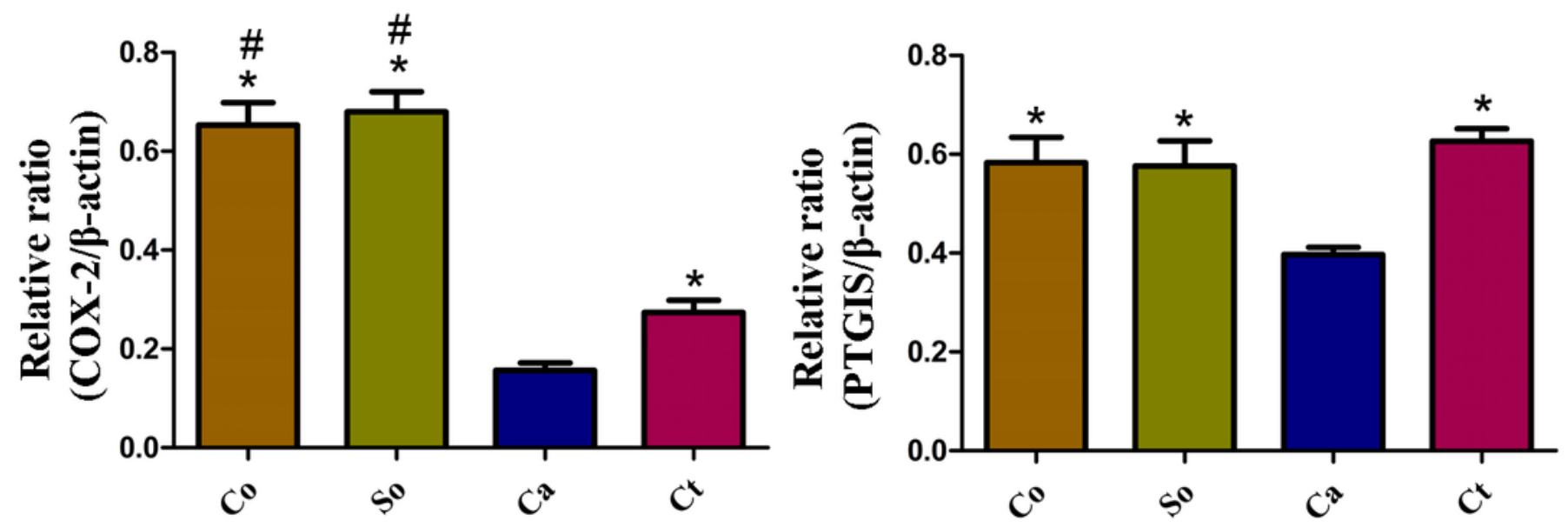